\title{
Fe-porphyrine complex atop of armchair graphene nanoribbon: multiplicity, coordination and potential energy surface of migration through ab initio PBC DFT framework
}

\author{
Iuliia Melchakova ${ }^{1}$, Artem Kuklin $^{2}$, and Paul Avramov ${ }^{1}$ \\ ${ }^{1}$ Kyungpook National University \\ ${ }^{2} \mathrm{KTH}$ Royal Institute of Technology School of Chemical Science
}

April 28, 2020

\begin{abstract}
The atomic structure, spin states of the interface based on iron-porphyrin and armchair graphene nanoribbon (FeP/AGNR) and potential energy surface of FeP atop of AGNR migration is investigated via DFT theory. The multiplicity of Fe ion in iron porphyrin for all possible types of coordination is determined as a triplet. It is estimated that FeP would place atop AGNR at the position where two Fe- $\mathrm{N}$ bonds are located above the $\mathrm{C}-\mathrm{C}$ bond, another two are located above $\mathrm{C}$ atoms. The barrier of migration of iron porphyrin complex atop of graphene armchair nanoribbon is found to be smaller the temperature factor, making the heterostructure to be in temperature equilibrium between different types of coordination of the iron porphyrin atop of graphene nanoribbon
\end{abstract}

Fe-porphyrine complex atop of armchair graphene nanoribbon: multiplicity, coordination and potential energy surface of migration through ab initio PBC DFT framework

Iuliia Melchakova ${ }^{1}$, Artem Kuklin ${ }^{2}$, Paul Avramov ${ }^{3}$

Correspondence to: Paul Avramov (e-mail: paul.veniaminovich@knu.ac.kr)

Iuliia Melchakova

Kyungpook National University, 80 Daehakro, Bukgu, Daegu, 41566, Korea

${ }^{2}$ Artem Kuklin

Division of Theoretical Chemistry and Biology, School of Engineering Sciences in Chemistry, Biotechnology and Health, KTH Royal Institute of Technology, 10691 Stockholm, Sweden

Department of science and innovations, Siberian Federal University, 79 Svobodny pr., Krasnoyarsk 660041

Kirensky Institute of Physics, FRC KSC SB RAS, 50 Akademgorodok, Krasnoyarsk, 660036,Russia

${ }^{3}$ Paul Avramov

Kyungpook National University, 80 Daehakro, Bukgu, Daegu, 41566, Korea

Introduction

The last 15 years the attention of material scientists is drawn to the world of $2 \mathrm{D}$ materials in particular to find candidates for spin-related applications. Heterostructure based on Me-porphyrin deposited on 2D materials can be found very promising because of variety of possible spin states and their low degeneracy with 
large distance between metallic centres preventing chemical interactions of the ions together with possible quantum entanglement of close to degeneracy localized spin states.

Graphene is one of the most popular planar material due to high charge carrier mobility, low hyperfine interaction as well as low intrinsic spin-orbital interaction. ${ }^{1}$ Due to its unique electronic structure (e.g. Dirac cone presence) graphene is a very promising material for spintronics applications. Taking into account the absence of spin-polarization, graphene can play a significant role as a part of novel heterostructures being the material that do not change the magnetic properties of other components allowing advanced transport properties. $^{2}$ Interfaces based on graphene/hBN

have mobilities and carrier inhomogeneities that are almost an order of magnitude higher than devices based on $\mathrm{SiO}_{2 .}{ }^{3,4}$ Due to simplicity of fabrication and opportunity to synthesize high-squared sheets, graphene is going to be the most popular material related to electronics in near future. ${ }^{5}$

A strong band gap dependence of narrow armchair graphene nanoribbons (AGNR) upon the edge effectswas demonstrated. ${ }^{6-8}$ It was found that the band gap decreases almost inversely upon the effective widths of the ribbons until reaching a vanishing value for $2 \mathrm{~nm}$ width nanoribbons. ${ }^{6,7,9,10}$ The AGNR lattices belong to three groups of width-dependent small, medium, and large energy band gaps with $\mathrm{N}=3 p+2,3 p+1$, and $3 p$, respectively, where $p$ is a positive integer equal to the number of carbon dimers oriented perpendicular to the ribbon. ${ }^{7,9,10}$ In fact, for $p=11$ (AGNR-11) the number of hexagonal rings perpendicular to the main axis of ribbon is equal to 9 . The AGNR-11 satisfactory reproduces the electronic structure of graphene (close to zero narrow band gap of $0.05 \mathrm{eV}$ with band structure resembles Dirac cone) in the vicinity of the Fermi level. ${ }^{6-8,10}$

Porphyrin belongs to a rich family of organic molecules that are of great interest in chemistry and physics. Due to presence of the active site in the centre of the molecule it can be filled by different transition metal (TM) ions. The TM-porphyrin complexes (TMP) demonstrate wide variety of spin and electronic properties. ${ }^{11,12}$ The $\mathrm{TM}$ ions (Fe, Mg, Co) implemented in porphyrin determine particular biological roles in living cells forming the hemes, the chlorophylls and the cobalamins. ${ }^{13}$ Graphene-based heterostructures with absorbed molecules with non-zero spin-polarized open shell electronic structure gain popularity in recent years. ${ }^{14,15}$ Spin-polarized molecules in contact with graphene constitute a tantalizing approach towards organic spin electronics because of the reduced conductivity mismatch at the interface ${ }^{15,16}$ and high catalytic activity $^{17}$. TMP/AGNR weakly bounded complexes may be considered as promising heterostructures for spin-related and quantum applications.

There are a lot of publications devoted to Me-porphyrin $\operatorname{properties}^{18,19}$, but the question about the migration pathway of the complexes on graphene surface is still open. Keeping in mind isotropic structure, flat potential energy profile and passivity of graphene surface, one can consider rotation and migration degrees of freedom of Me-porphyrin complexes on it. This paper is aimed to shed a light at the migration and rotation pathways of Fe-porphyrin (FeP) at AGNR surface, to determine the spin states of Fe ion in FeP complex (FeP/AGNR), and to locate its favourable coordinations relatively AGNR and to characterize migration potential energy pathways.

\section{Methods}

The electronic structure calculations of heterostructures designed by deposition of porphyrin and ironporphyrin atop of AGNR-11 (Figure 1), the angle-dependent orientation of the porphyrin and the potential energy surface of Fe-porphyrin migration atop AGNR-11 were performed using Long-Corrected Generalized Gradient Approximation HSE functional ${ }^{20}$ along with Gaussian-type basis set ${ }^{21}$ (6-21G on hydrogen and 6-31G/ on carbon) and Periodic Boundary Conditions (PBC LC GGA/HSE DFT) implemented in Gaussian 09. ${ }^{22}$ To optimize AGNR-11 and calculate energy profiles, 128 points in k-space for the Brillouin zone integration scheme was used. The self-consistency threshold of $10^{-6}$ Hartree for the total energy was used during SCF iterations. All geometries were optimized without symmetry restrictions to avoid converging to higher energy solution wave functions imposed by preliminarily determined symmetry restrictions. 
In perpendicular to the main nanoribonn axis, the AGNR-11 contains 9 hexagonal carbon rings, which perfectly matches the dimension of porphyrin molecule (Figure 1). To satisfy the dimension of the porphyrin molecule and avoid any physical and chemical interactions between the porphyrin molecules in neighbouring PBC images, the length of the AGNR-11 supercell was chosen equal to 6 hexagonal carbon rings (Figure 1 ). In order to avoid dangling bonds and keep puresp ${ }^{2}$ nature of all carbon-carbon connections, the edge carbon atoms are passivated by hydrogen atoms.

To localize global and local minima, several initial configurations of porphyrin and Fe-porphyrin were considered. In particular, four different coordinations of porphyrin atop AGNR-11 (P/AGNR) were considered: carbon atoms of AGNR are located under N-H bond of porphyrin (O1), C-C bond of AGNR is located under $\mathrm{N}-\mathrm{H}$ bond of porphyrin (O2), carbon atom of AGNR is located under $\mathrm{N}$ atom of porphyrin (O3), and carbon atoms of AGNR are located under N-H bond of porphyrin like (O1) but turned at $45^{0}$ around the normal to plane (O4) (Figure S1). For FeP/AGNR heterostructures 3 different types of rotational coordinations were considered: For the first coordination, two Fe-N bonds were located above $\mathrm{C}-\mathrm{C}$ bond of ARNG, another two were located above $\mathrm{C}$ atoms $(\mathrm{C} 1)$; In second coordination all Fe- $\mathrm{N}$ bonds were located above $\mathrm{C}$ atoms $(\mathrm{C} 2)$; And finally, the third coordination is characterized geometrically like $(\mathrm{C} 1)$ turned at $30^{\circ}$ around the normal direction $(\mathrm{C} 2)$.

The standard notations for hexagon orientation were used $\left(\eta_{\mathrm{x}}\right)^{23}$ to distinguish different mutual coordination of $\mathrm{Fe}$ ion of $\mathrm{FeP}$ complex to the AGNR-11 substrate at the migration pathway determination. In particular, 3 positions of FeP relatively to AGNR-11 were set, namely $\eta_{1}, \eta_{2}, \eta_{6}$, which correspond to coordination of Fe ion atop of single carbon atom of AGNR, the centre of C-C bond and centre of carbon hexagon, respectively.

Following reasonable assumption, the migration has no impact on details of atomic structure of FeP complex, but do have the impact on the distance between complex and AGNR in normal direction. The procedure of the PES determination was consisted on restrained optimization of constituting fragments (Fe-porphyrine and AGNR-11) only along z direction on $\eta_{1}, \eta_{2}$, and $\eta_{6}$ pathways.

\section{Results and discussion}

Structural characterization

Relative energies for different angles of porphyrin molecule rotation atop AGNR are presented in Table 1. The O2 coordination was determined as energetically favourable (Figure 1) with very low $(0.15-0.33 \mathrm{kcal} / \mathrm{mol})$ relative energies of $\mathrm{O} 1, \mathrm{O} 3$ and $\mathrm{O} 4$ coordinations. Comparison of the relative energies in temperature units (75-166K) to the temperature factor (Table 1) demonstrate that finite temperature fluctuations can initiate the rotation of porphyrin on AGNR.

Table 1. Relative energies for different angles of porphyrin rotation atop of AGNR-11 in respect to O2 configuration $(\Delta \mathrm{E}=0 \mathrm{kcal} / \mathrm{mol})$.

\begin{tabular}{llll}
\hline Structure & $\Delta \mathrm{E}, \mathrm{kcal} / \mathrm{mol}$ & $\Delta \mathrm{E}, \mathrm{K}$ & Interlayer distance, $\AA$ \\
\hline Porphyrin/AGNR (O1) & 0.15 & 75.5 & 3.72 \\
Porphyrin/AGNR (O2) & 0.00 & 0.00 & 3.77 \\
Porphyrin/AGNR (O3) & 0.33 & 166.1 & 3.72 \\
Porphyrin/AGNR (O4) & 0.28 & 140.9 & 3.77 \\
\hline
\end{tabular}




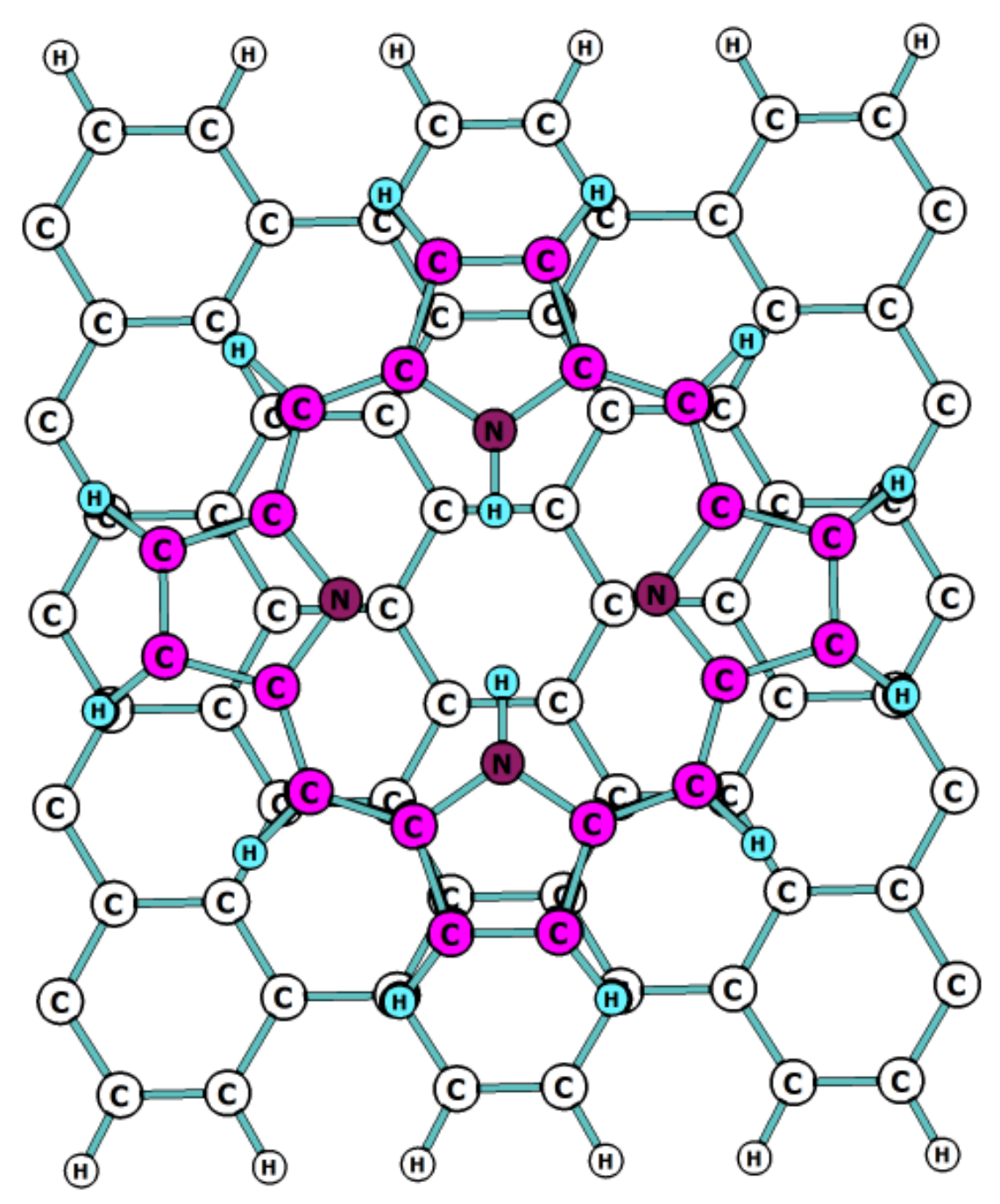

Figure 1. Atomic structure of $\mathrm{P} / \mathrm{AGNR}-11$ complex in $\mathrm{O} 2$ coordination. The distance between the fragments is $3.7 \AA$. Carbon atoms of AGNR-11 are denoted in white, carbon atoms of $\mathrm{P}$ are denoted in purple, hydrogen atoms are denoted in blue.

The relative energies of spin states of FeP/AGNR are presented in Table 2. It was found that among 3 possible spin states (singlet, triplet, quintet), the Fe $d^{6}$ triplet state with two unpaired electrons in $d_{y z}$ and $d_{x z}$ orbitals (Figure 2) is energetically preferable, which coincides with previous theoretical data ${ }^{19}$ at GGA PBE level of theory. The energy differences between triplet and singlet and triplet and quintet states in infrared energy region (40 and $28 \mathrm{kcal} / \mathrm{mol}$, respectively) directly indicate possible optical activity and spin-flip transitions in FeP complex atop AGNR.

Table 2. Relative energies of the FeP complex in different spin states.

\begin{tabular}{ll}
\hline Multiplicity & $\Delta \mathrm{E}, \mathrm{kcal} / \mathrm{mol}$ \\
\hline Singlet $(\mathrm{M}=1)$ & 40.00 \\
Triplet $(\mathrm{M}=3)$ & 0.00 \\
Quintet $(\mathrm{M}=5)$ & 28.00 \\
\hline
\end{tabular}




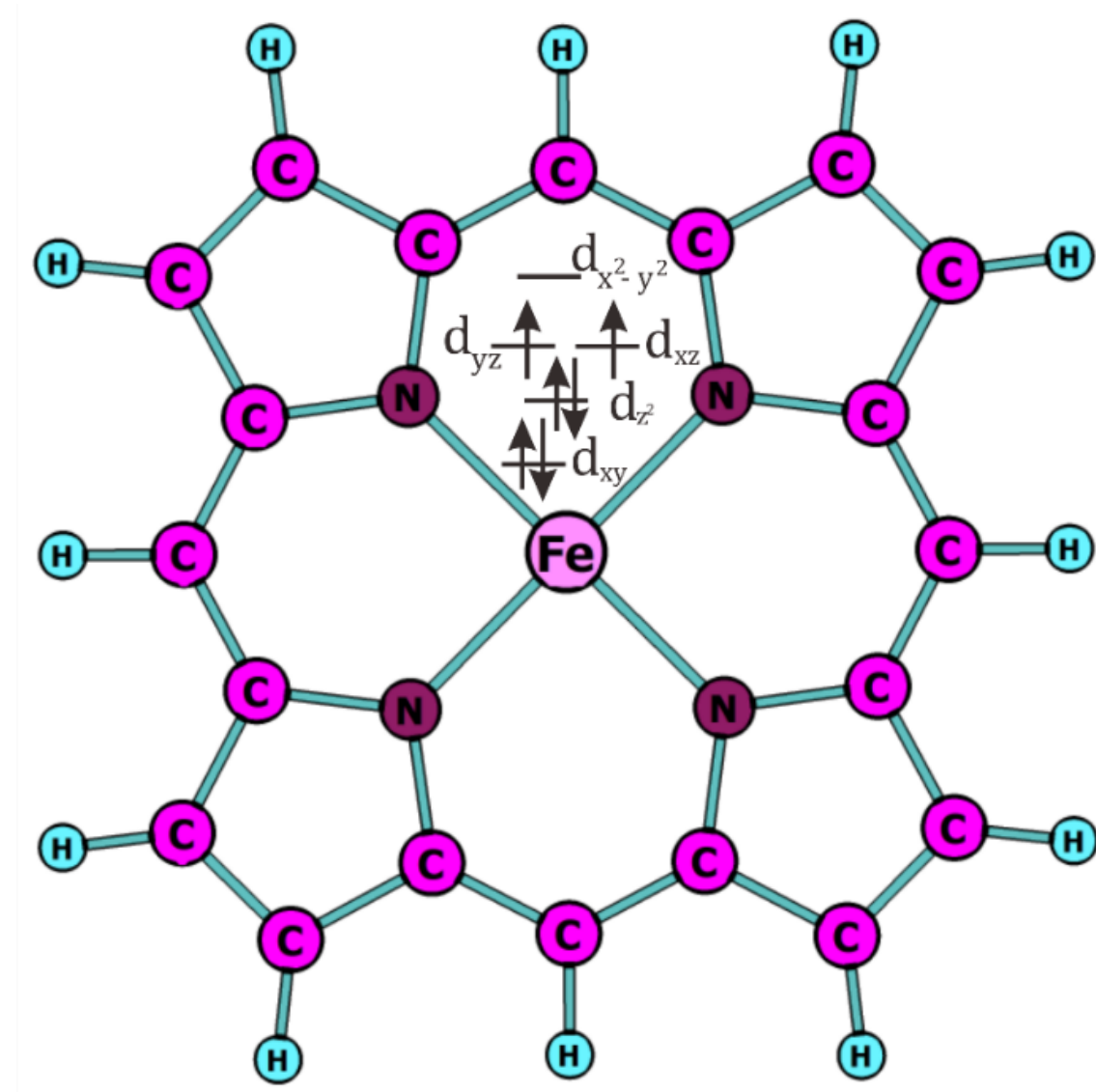

Figure 2. Atomic structure and Fe $d^{6}$ electron configuration of free-standing Fe-Porphyrin complex in triplet state ${ }^{24,25}$. Fe $d^{6}$ electronic configuration with two unpaired electrons on $d_{y z}$ and $d_{x z}$ is shown with arrows.

At PBC LC GGA/HSE DFT level of theory C1, C2 and C3 configurations of FeP/AGNR complex were localized (Table 3) with global minimum at $\mathrm{C} 1$ with the differences between coordinations caused by rotational angle of FeP relatively to AGNR (Figure S2). At the same time, singlet/triplet states were considered to estimate the most probable spin state in the case of FeP/AGNR heterostructure Table S1 and it was found that the triplet state is energetically preferable for FeP/AGNR as well.

Table 3. Relative energies of Fe(triplet)P/AGNR complex in different configurations.

\begin{tabular}{lll}
\hline Structure & $\Delta \mathrm{E}, \mathrm{kcal} / \mathrm{mol}$ & Interlayer distance, $\AA$ \\
\hline Fe-Porphyrin/AGNR (C1) & 0.000 & 3.67 \\
Fe-Porphyrin/AGNR (C2) & 13.969 & 3.69 \\
Fe-Porphyrin/AGNR (C3) & 1.104 & 3.72 \\
\hline
\end{tabular}

At the HSE/6-31G level of theory, the $\mathrm{C} 1$ configuration is energetically preferable with relative energies of C2 and C3 configurations equal to 1.104 and $13.969 \mathrm{kcal} / \mathrm{mol}$, respectively (Table 3). The energy difference value is found much higher the temperature factor for $\mathrm{C} 2(7029.5 \mathrm{~K})$ and proportional for $\mathrm{C} 3(555.5 \mathrm{~K})$, which limits rotation of iron porphyrin on top of AGNR without external optical excitation.

Migration pathway 
To analyze the migration pathway $\eta_{1}, \eta_{2}$, and $\eta_{6}$ coordinations of FeP to AGNR-11 were considered (Figure S3) and the relative energies are demonstrated in Table S3. At HSE/6-31G level of theory $\eta_{6}$ position is the global minimum of $\mathrm{FeP} / \mathrm{AGNR}$ with relative energies of $\eta_{1}$ and $\eta_{2}$ equal to 0.37 and $0.28 \mathrm{kcal} / \mathrm{mol}$. Due to small values of relative energies of both $\eta_{1}$ and $\eta_{2}$ positions, the presence of the thermal equilibrium of all coordinations can be assumed at normal conditions according Arrhenius distribution. To analyse the energy barriers of $\eta^{\prime}$ 's transitions, the $\eta_{2}-\eta_{1}-\eta_{6}$ migration pathway was calculated. The relative energies of critical points on potential energy curve are presented in Figure 3.

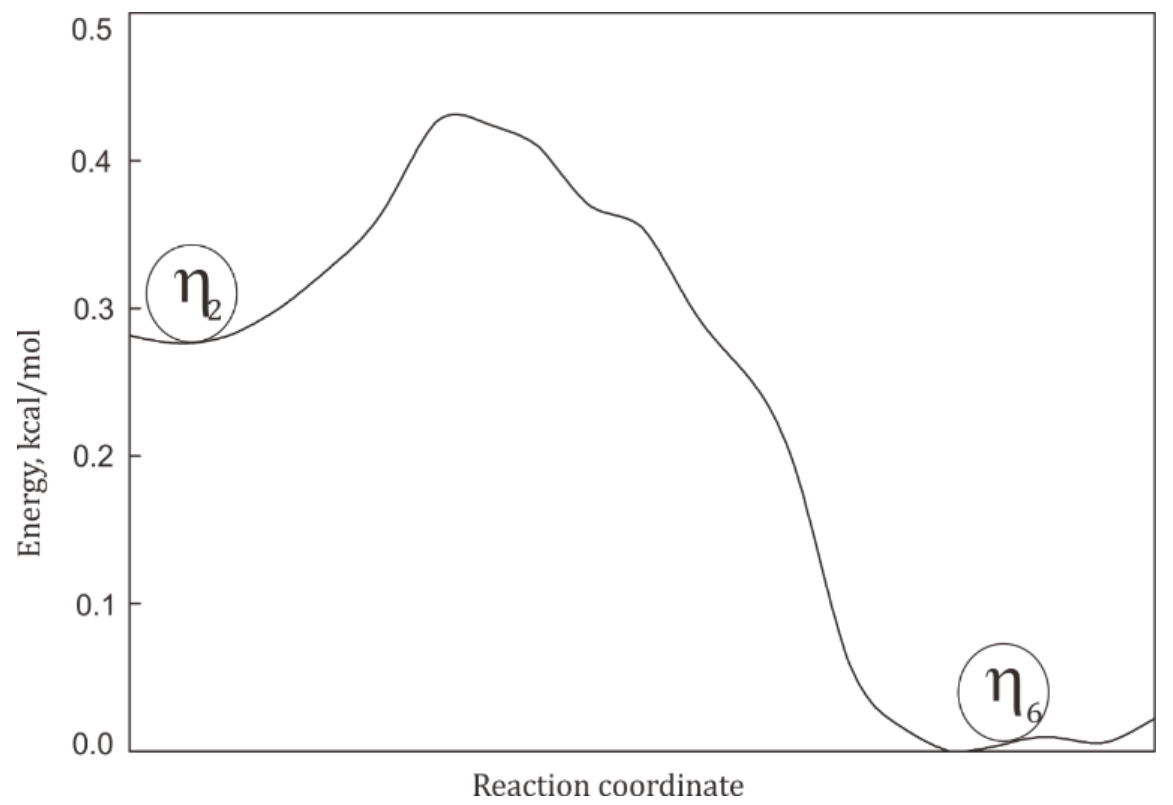

Figure 3. The potential energy curve along $\eta_{2^{-}} \eta_{1^{-}} \eta_{6}$ migration pathway of FeP complex atop AGNR-11. The positions of local $\left(\eta_{2}\right)$ and global $\left(\eta_{6}\right)$ are denoted in circles.

Two local and global minima were localized at $\eta_{2}$ and $\eta_{6}$ coordinations, respectively with forward potential and reversed barriers between $\eta_{2}$ and $\eta_{6}$ equal to $0.15 \mathrm{kcal} / \mathrm{mol}(75 \mathrm{~K})$ and $0.45 \mathrm{kcal} / \mathrm{mol}(226 \mathrm{~K})$, respectively. Due to small barrier values it is reasonable to suppose chemical equilibrium between coordinations with 39 $\%$ and to $61 \%$ Arrhenius weights for $\eta_{2}$ and $\eta_{6}$ coordinations, respectively.

\section{Conclusions}

Using PBC LC GGA/HSE DFT electronic structure calculations, the atomic structure of P/AGNR-11 and Fe-P/AGNR-11 heterostructures and spin states of Fe-P/AGNR-11was considered. It was found that both porphyrin and Fe-porphyrin are bound with AGNR substrate by weak disperse interactions. The rotational degree of freedom of porphyrin on AGNR substrate and the FeP migration atop ANGR was found thermal active, i.e. they can be initiated by temperature fluctuations. Keeping this fact in mind it reasonable to suppose thermal equilibrium between different coordination. In the same time, the rotation of $\mathrm{FeP}$ on AGNR and spin-flip transitions were considered as optical active, i.e. they can be initiated by external excitations. It was found that the Fe-Porphyrin complex in triplet state is coordinated to AGNR in $\eta_{6}$ position with $\mathrm{C} 1$ rotational angle. The potential barriers between $\eta_{2}$, and $\eta_{6}$ coordinations are lower or comparable with temperature factor with $39 \%$ and $61 \%$ Arrhenius populations.

\section{Funding information}

The research was supported by the Russian Science Foundation (Project No.19-73-10015). Korean team was supported by BK21+. 
Keywords: Fe-porphyrin, AGNR, DFT PBC, spin states, potential energy curve

\section{References and Notes}

1. A. K. Geim and K. S. Novoselov, Nat. Mater., 2007, 6, 183-191.

2. P. V. Avramov, P. B. Sorokin, A. A. Kuzubov, S. Sakai, S. Entani and H. Naramoto, Aust. J. Chem., 2016, 69, 753-758.

3. C. R. Dean, A. F. Young, I. Meric, C. Lee, L. Wang, S. Sorgenfrei, K. Watanabe, T. Taniguchi, P. Kim, K. L. Shepard and J. Hone, Boron nitride substrates for high quality graphene electronics, .

4. K. Zollner, M. Gmitra and J. Fabian, Phys. Rev. B, 2019, 99, 125151.

5. P. Bhauriyal, G. Bhattacharyya, K. S. Rawat and B. Pathak, J. Phys. Chem. C, 2019, 123, 3959-3967.

6. M. Y. Han, B. Özyilmaz, Y. Zhang and P. Kim, Phys. Rev. Lett., 2007, 98, 206805.

7. V. Barone, O. Hod and G. E. Scuseria, Nano Lett., 2006, 6, 2748-2754.

8. S. Dutta, S. Lakshmi and S. K. Pati, Phys. Rev. B - Condens. Matter Mater. Phys., 2008, 77, 073412.

9. A. Nduwimana and X.-Q. Wang, ACS Nano, 2009, 3, 1995-1999.

10. Y. W. Son, M. L. Cohen and S. G. Louie, Phys. Rev. Lett., 2006, 97.

11. H. Hirao, S. Shaik and P. M. Kozlowski, J. Phys. Chem. A, 2006, 110, 6091-6099.

12. J. F. Lefebvre, D. Leclercq, J. P. Gisselbrecht and S. Richeter, European J. Org. Chem., 2010, 2010, 1912-1920.

13. J. Fyrestam, N. Bjurshammar, E. Paulsson, A. Johannsen and C. Östman, Anal. Bioanal. Chem., 2015, 407, 7013-7023.

14. M. Coroş, F. Pogăcean, L. Măgeruşan, M. C. Roşu, A. S. Porav, C. Socaci, A. Bende, R. I. Stefan-van Staden and S. Pruneanu, Sensors Actuators, B Chem., 2018.

15. S. Bhandary, S. Ghosh, H. Herper, H. Wende, O. Eriksson and B. Sanyal, .

16. P. Kratzer, S. A. Tawfik, X. Y. Cui and C. Stampfl, RSC Adv., 2017, 7, 29112-29121.

17. J. Choi, P. Wagner, R. Jalili, J. Kim, D. R. MacFarlane, G. G. Wallace and D. L. Officer, Adv. Energy Mater., 2018, 8, 1801280.

18. T. Higashino and H. Imahori, Dalt. Trans., 2015, 44, 448-463.

19. A. R. Groenhof, M. Swart, A. W. Ehlers and K. Lammertsma, 2005.

20. J. Heyd, G. E. Scuseria and M. Ernzerhof, J. Chem. Phys., 2003, 118, 8207-8215.

21. P. V. Avramov, S. Sakai, S. Entani, Y. Matsumoto and H. Naramoto, Chem. Phys. Lett., 2011, 508, 86-89.

22. M. J. Frisch, G. W. Trucks, H. B. Schlegel, G. E. Scuseria, M. A. Robb, J. R. Cheeseman, G. Scalmani, V. Barone, G. A. Petersson, H. Nakatsuji, X. Li, M. Caricato, A. V Marenich, J. Bloino, B. G. Janesko, R. Gomperts, B. Mennucci, H. P. Hratchian, J. V Ortiz, A. F. Izmaylov, J. L. Sonnenberg, D. WilliamsYoung, . Ding, F. Lipparini, F. Egidi, J. Goings, B. Peng, A. Petrone, T. Henderson, D. Ranasinghe, V. G. Zakrzewski, J. Gao, N. Rega, G. Zheng, W. Liang, M. Hada, M. Ehara, K. Toyota, R. Fukuda, J. Hasegawa, M. Ishida, T. Nakajima, Y. Honda, O. Kitao, H. Nakai, T. Vreven, K. Throssell, J. A. Montgomery Jr., J. E. Peralta, F. Ogliaro, M. J. Bearpark, J. J. Heyd, E. N. Brothers, K. N. Kudin, V. N. Staroverov, T. A. Keith, R. Kobayashi, J. Normand, K. Raghavachari, A. P. Rendell, J. C. Burant, S. S. Iyengar, J. Tomasi, M. Cossi, J. M. Millam, M. Klene, C. Adamo, R. Cammi, J. W. Ochterski, R. L. Martin, K. Morokuma, O. Farkas, J. B. Foresman and D. J. Fox, 2016. 
23. P. Avramov, H. Naramoto, S. Sakai, K. Narumi, V. Lavrentiev and Y. Maeda, J. Phys. Chem. A, 2007, 111, 2299-2306.

24. F. A. Walker and U. Simonis, in Encyclopedia of Inorganic Chemistry, John Wiley \& Sons, Ltd, Chichester, UK, 2006.

25. C. Rovira, K. Kunc, J. Hutter, P. Ballone and M. Parrinello, J. Phys. Chem. A, 1997, 101, 8914-8925.

\section{GRAPHICAL ABSTRACT}

\section{Iuliia Melchakova, Artem Kuklin, Paul Avramov}

Fe-porphyrine complex atop of armchair graphene nanoribbon: multiplicity, coordination and potential energy surface of migration through ab initio PBC DFT framework

Heterostructure based on Fe-porphyrine molecule and graphene armchair nanoribbon stacked pairwise was investigated. It possess special properties in migration and rotation processes: low degeneracy of different spin-states of Fe center and small value of the $\eta_{2}-\eta_{6}$ transition that define of great interest for technological applications.

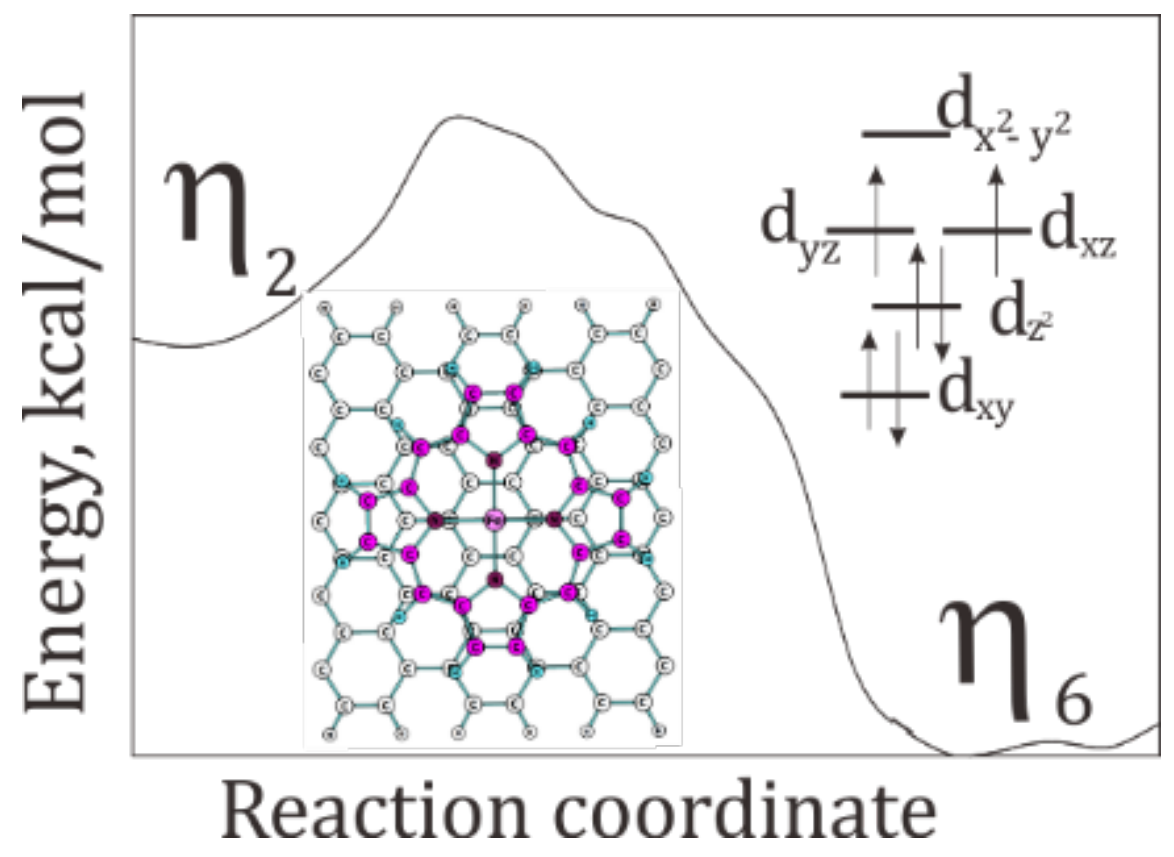

